\title{
Extended Anticoagulant Treatment with Full- or Reduced-Dose Apixaban in Patients with Cancer-Associated Venous Thromboembolism: Rationale and Design of the API-CAT Study
}

\author{
Isabelle Mahé ${ }^{1,2,3,40}$ Giancarlo Agnelli ${ }^{5}$ Cihan Ay ${ }^{6}$ Aristotelis Bamias ${ }^{70}$ Cecilia Becattini ${ }^{5}$ \\ Marc Carrier $^{8}$ Céline Chapelle ${ }^{9,10}$ Alexander T. Cohen ${ }^{11}$ Philippe Girard ${ }^{12}$ Menno V. Huisman ${ }^{13}$ \\ Frederikus A. Klok ${ }^{13}$ Juan J. López-Núñez ${ }^{14,15}$ Anthony Maraveyas ${ }^{16}$ Didier Mayeur $^{17}$ Olivier Mir $^{18}$ \\ Manuel Monreal ${ }^{14,15,19}$ Marc Righini ${ }^{20}$ Charles M. Samama ${ }^{21}$ Kostas Syrigos $^{22}$ Sebastian Szmit ${ }^{23}$ \\ Adam Torbicki $^{23}$ Peter Verhamme ${ }^{24}$ Eric Vicaut ${ }^{25}$ Tzu-Fei Wang ${ }^{8}$ Guy Meyer $2,4,26$
}

Silvy Laporte L,9,10 $^{4,10}$

1 Service de Médecine Interne, Hôpital Louis Mourier, AP-HP, Colombes, France

2 Université de Paris, Paris, France

${ }^{3}$ Innovative Therapies in Haemostasis, INSERM UMR_S1140, Paris, France

${ }^{4}$ INNOVTE-FCRIN, Saint-Etienne, France

5 Internal Vascular and Emergency Medicine - Stroke Unit, University of Perugia, Perugia, Italy

${ }^{6}$ Clinical Division of Haematology and Haemostaseology, Department of Medicine I, Comprehensive Cancer Center Vienna, Medical University of Vienna, Vienna, Austria

7 2nd Propaedeutic Department of Medicine, ATTIKON University Hospital, National \& Kapodistrian University of Athens, Athens, Greece

${ }^{8}$ Department of Medicine, Ottawa Hospital Research Institute, University of Ottawa, Ottawa, Ontario, Canada

${ }^{9}$ Unité de Recherche Clinique Innovation et Pharmacologie, CHU de Saint-Etienne, Saint-Etienne, France

10 SAINBIOSE INSERM U1059, Université Jean Monnet, Université de Lyon, Saint-Etienne, France

${ }^{11}$ Guy's and St. Thomas' NHS Foundation Trust Hospital, King's College London, London, United Kingdom

12 Département de Pneumologie, Institut Mutualiste Montsouris, Paris, France

${ }^{13}$ Department of Medicine - Thrombosis and Hemostasis, Leiden University Medical Center, Leiden, The Netherlands

${ }^{14}$ Department of Internal Medicine, Hospital Universitari Germans Trias i Pujol, Badalona, Spain

Thromb Haemost 2022;122:646-656.
Address for correspondence Isabelle Mahé, MD, PhD, Chef de Service, Hôpital Louis Mourier, Service de Médecine Interne, Université de Paris, AP-HP, 178 rue des Renouillers, 92700 Colombes, France (e-mail: isabelle.mahe@aphp.fr).

${ }^{15}$ Department of Medicine, Universitat Autònoma de Barcelona, Barcelona, Spain

16 Queen's Centre for Oncology and Haematology, Hull University Teaching Hospitals NHS Trust, Hull York Medical School, Cottingham, Hull, United Kingdom

${ }^{17}$ Centre Georges-Francois Leclerc, Dijon, France

18 Institut Gustave-Roussy, Paris, France

19 Universidad Católica de Murcia, Murcia, Spain

20 Division of Angiology and Hemostasis, Faculty of Medicine, Geneva University Hospitals, Geneva, Switzerland

${ }^{21}$ Intensive Care and Perioperative Medicine, Department of Anaesthesia, Hôpital Cochin, GHU AP-HP Centre-Université de Paris, Paris, France

22 Department of Medicine, Sotiria General Hospital, Athens School of Medicine, National \& Kapodistrian University, Athens, Greece

${ }^{23}$ Departments of Pulmonary Circulation, Thromboembolic Diseases, and Cardiology, Centre of Postgraduate Medical Education, European Health Centre, Otwock, Poland

${ }^{24}$ Vascular Medicine and Haemostasis, University Hospitals Leuven, Leuven, Belgium

${ }^{25}$ URC Lariboisière - Saint-Louis, AP-HP, Université de Paris, Paris, France

${ }^{26}$ Hôpital Européen Georges Pompidou, APHP, Sorbonne Paris Cité, Paris, France received

May 27, 2021

accepted after revision

July 8,2021

published online

September 17, 2021
DOI https://doi.org/ 10.1055/a-1647-9896. ISSN 0340-6245. (c) 2021. The Author(s).

This is an open access article published by Thieme under the terms of the Creative Commons Attribution-NonDerivative-NonCommercial-License, permitting copying and reproduction so long as the original work is given appropriate credit. Contents may not be used for commercial purposes, or adapted, remixed, transformed or built upon. (https://creativecommons.org/ licenses/by-nc-nd/4.0/)

Georg Thieme Verlag KG, Rüdigerstraße 14, 70469 Stuttgart, Germany 


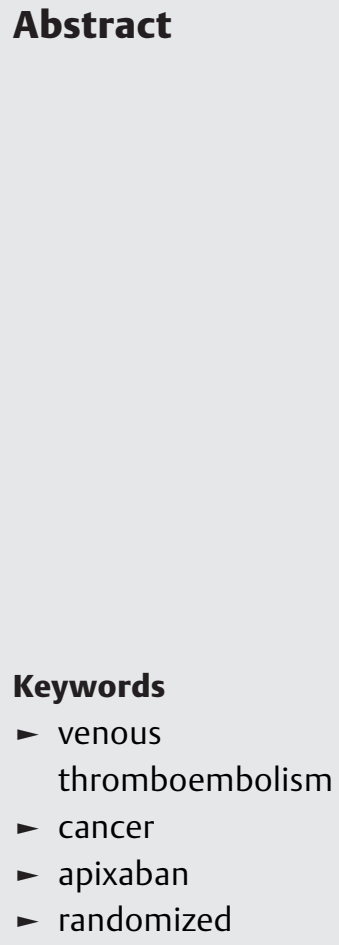

Abstract

Cancer-associated thrombosis (CT) is associated with a high risk of recurrent venous thromboembolic (VTE) events that require extended anticoagulation in patients with active cancer, putting them at risk of bleeding. The aim of the API-CAT study (NCT03692065) is to assess whether a reduced-dose regimen of apixaban ( $2.5 \mathrm{mg}$ twice daily [bid]) is noninferior to a full-dose regimen of apixaban ( $5 \mathrm{mg}$ bid) for the prevention of recurrent VTE in patients with active cancer who have completed $\geq 6$ months of anticoagulant therapy for a documented index event of proximal deep-vein thrombosis and/or pulmonary embolism. API-CAT is an international, randomized, parallel-group, double-blind, noninferiority trial with blinded adjudication of outcome events. Consecutive patients are randomized to receive apixaban 2.5 or $5 \mathrm{mg}$ bid for 12 months. The primary efficacy outcome is a composite of recurrent symptomatic or incidental VTE during the treatment period. The principal safety endpoint is clinically relevant bleeding, defined as a composite of major bleeding or nonmajor clinically relevant bleeding. Assuming a 12-month incidence of the primary outcome of $4 \%$ with apixaban and an upper limit of the two-sided 95\% confidence interval of the hazard ratio $<2.0,1,722$ patients will be randomized, assuming an up to $10 \%$ loss in total patient-years $(\beta=80 \% ; \alpha$ one-sided $=0.025)$. This trial has the potential to demonstrate that a regimen of extended treatment for patients with $\mathrm{CT}$ beyond an initial 6 months, with a reduced apixaban dose, has an acceptable risk of recurrent VTE recurrence and decreases the risk of bleeding.

\section{Introduction}

Venous thromboembolism (VTE), encompassing deep-vein thrombosis (DVT) and pulmonary embolism (PE), is a common complication of cancer, and is often referred to as cancer-associated thrombosis (CT). CT is associated with a high risk of VTE recurrence, a life-threatening complication that requires long-term anticoagulation for as long as the underlying cancer remains active. ${ }^{1}$ In contrast to stable figures for VTE incidence in the general population without cancer, the incidence and prevalence of VTE have been increasing in patients with cancer, ${ }^{2}$ and can be explained by many factors: the rising incidence and/or prevalence of cancer, longer survival of patients with metastatic cancer, greater use of prothrombotic drugs and catheters, and progress and improvement in the performance of diagnostic tests for VTE. ${ }^{3,4}$ Furthermore, progress in anticancer treatments and supportive care has considerably changed the prognosis for patients with cancer, resulting in an increasing number of longer-surviving patients, whatever the site of cancer., ${ }^{2,4-9}$ Consequently, up to $60 \%$ of patients with cancer are still alive 6 months after the index VTE event. ${ }^{10,11}$ The optimal treatment beyond the first 6 months of treatment for CT-a disease with considerable economic impact ${ }^{12,13}$-is unknown, but lies in the prevention of recurrent VTE while avoiding excess bleeding. ${ }^{12,13}$

After 6 months of anticoagulant treatment, the risk of VTE recurrence is lower than during the first 6 months, but is still considerable in patients with CT. Indeed, an observational study performed between 2001 and 2011 by the United Kingdom Clinical Practice Research Datalink reported an incidence rate for recurrent VTE of 22.1 per 100 patientyears (95\% confidence interval $[\mathrm{CI}]$ : 19.9-24.4) during the first 6 months and of 7.9 per 100 patient-years (95\% CI: 6.29.2) beyond 6 months and up to 12 months. ${ }^{14}$ In addition, in a cohort of consecutive patients with $\mathrm{CT}$, the risk of VTE recurrence was low in patients cured of cancer but remained high in those who stopped anticoagulant treatment despite active cancer (19 per 100 patient-years). ${ }^{15}$ Based on these results and the known persisting risk of VTE for as long as the cancer remains active, current guidelines suggest continuing anticoagulant therapy with no scheduled stop date as long as the cancer is active and/or anticancer treatment is ongoing. ${ }^{16-19}$ However, continuing anticoagulant therapy in the context of active cancer is associated with a substantial risk of bleeding, as documented in prospective cohorts. ${ }^{20,21}$ In addition, the clinical course may differ according to the site of cancer, in terms of VTE recurrence and bleeding risks. ${ }^{22}$ Therefore, the optimal therapeutic option beyond 6 months is not clearly defined.

Although subcutaneous injections appear to be well accepted by most patients during the first weeks of treatment, it is difficult to continue treatment using this route of administration indefinitely. ${ }^{23}$ Several randomized controlled trials have compared direct oral anticoagulants (DOACs) and low-molecular-weight heparins (LMWHs) for the initial treatment of patients with CT up to 6 months (- Table 1)..$^{24-27}$ Overall, the rate of recurrent VTE is slightly lower in patients on DOACs, but at the cost of a higher rate of major and/or clinically relevant nonmajor bleeding. ${ }^{24,25,28}$ Considering the reduced rate of VTE recurrence and the persisting risk of bleeding after the first 6 months of anticoagulant 
Table 1 Randomized controlled trials comparing DOACs with LMWHs during the first 6 months of anticoagulation in the treatment of VTE in patients with cancer

\begin{tabular}{|c|c|c|c|c|c|c|c|}
\hline \multirow[t]{2}{*}{ Trial } & \multirow[t]{2}{*}{ NCT number } & \multirow{2}{*}{$\begin{array}{l}\text { Sample } \\
\text { size }(n)\end{array}$} & \multirow[t]{2}{*}{ Study design } & \multirow{2}{*}{$\begin{array}{l}\text { DOAC } \\
\text { duration }\end{array}$} & \multirow{2}{*}{$\begin{array}{l}\text { Comparator } \\
\text { duration }\end{array}$} & \multicolumn{2}{|l|}{ Results } \\
\hline & & & & & & DOAC & Comparator \\
\hline HOKUSAI $^{24}$ & NCT02073682 & 1050 & $\begin{array}{l}\text { PROBE, } \\
\text { noninferiority }\end{array}$ & $\begin{array}{l}\text { Edoxaban } \\
6-12 \text { months }\end{array}$ & $\begin{array}{l}\text { Dalteparin } \\
6-12 \text { months }\end{array}$ & $\begin{array}{l}\text { rVTE: } 7.9 \% \\
\text { MB: } 6.9 \% \\
\text { CRB: } 18.6 \%\end{array}$ & $\begin{array}{l}\text { rVTE: } 11.3 \% \\
\text { MB: } 4.0 \% \\
\text { CRB: } 13.9 \%\end{array}$ \\
\hline SELECT-D ${ }^{25}$ & - & 406 & PROBE & $\begin{array}{l}\text { Rivaroxaban } \\
6 \text { months }\end{array}$ & $\begin{array}{l}\text { Dalteparin } \\
6 \text { months }\end{array}$ & $\begin{array}{l}\text { rVTE: } 3.9 \% \\
\text { MB: } 5.4 \% \\
\text { CRB: } 17.7 \%\end{array}$ & $\begin{array}{l}\text { rVTE: } 8.9 \% \\
\text { MB: } 3.0 \% \\
\text { CRB: } 6.4 \%\end{array}$ \\
\hline ADAM-VTE $^{26}$ & NCT02585713 & 300 & Pilot, PROBE & $\begin{array}{l}\text { Apixaban } \\
6 \text { months }\end{array}$ & $\begin{array}{l}\text { Dalteparin } \\
6 \text { months }\end{array}$ & $\begin{array}{l}\text { rVTE: } 0.7 \% \\
\text { MB: } 0.0 \% \\
\text { CRB: } 6.2 \%\end{array}$ & $\begin{array}{l}\text { rVTE: } 6.3 \% \\
\text { MB: } 1.4 \% \\
\text { CRB: } 6.3 \%\end{array}$ \\
\hline CARAVAGGIO ${ }^{27}$ & NCT03045406 & 1170 & $\begin{array}{l}\text { PROBE, } \\
\text { noninferiority }\end{array}$ & $\begin{array}{l}\text { Apixaban } \\
6 \text { months }\end{array}$ & $\begin{array}{l}\text { Dalteparin } \\
6 \text { months }\end{array}$ & $\begin{array}{l}\text { rVTE: } 5.6 \% \\
\text { MB: } 3.8 \% \\
\text { CRB: } 12.2 \%\end{array}$ & $\begin{array}{l}\text { rVTE: } 7.9 \% \\
\text { MB: } 4.0 \% \\
\text { CRB: } 9.7 \%\end{array}$ \\
\hline $\begin{array}{l}\text { CASTA-DIVA } \\
\text { (completed) }\end{array}$ & NCT02746185 & 159 & PROBE & $\begin{array}{l}\text { Rivaroxaban } \\
3 \text { months }\end{array}$ & $\begin{array}{l}\text { Dalteparin } \\
3 \text { months }\end{array}$ & - & - \\
\hline $\begin{array}{l}\text { CONKO-011 } \\
\text { (ongoing) }\end{array}$ & NCT02583191 & 450 & Open label & $\begin{array}{l}\text { Rivaroxaban } \\
3 \text { months }\end{array}$ & $\begin{array}{l}\text { LMWH } \\
3 \text { months }\end{array}$ & - & - \\
\hline $\begin{array}{l}\text { CANVAS } \\
\text { (ongoing) }\end{array}$ & NCT02744092 & 811 & Open label & $\begin{array}{l}\text { Any DOAC } \\
6 \text { months }\end{array}$ & $\begin{array}{l}\mathrm{LMWH} \pm \mathrm{VKA} \\
6 \text { months }\end{array}$ & - & - \\
\hline
\end{tabular}

Abbreviations: CRB, clinically relevant bleeding (major bleeding and/or clinically relevant nonmajor bleeding); DOAC, direct oral anticoagulant; LMWH, low-molecular-weight heparin; MB, major bleeding; PROBE, prospective randomized open blinded endpoint; rVTE, recurrent venous thromboembolic event; VTE, venous thromboembolic event.

treatment, ${ }^{20,21}$ a reduced dose of DOAC for the long-term (>6 months) management of patients with $\mathrm{CT}$, as proposed to VTE patients without cancer, ${ }^{29,30}$ appears to be an appealing therapeutic option. We designed the API-CAT study, a prospective, double-blind, randomized trial comparing a reduced-dose regimen of apixaban $(2.5 \mathrm{mg}$ twice daily [bid]) with a full-dose regimen of apixaban ( $5 \mathrm{mg}$ bid) for the long-term prevention of recurrent VTE in patients with CT.

Since the design and start of the API-CAT study, the results of the CARAVAGGIO trial and the ADAM-VTE study, as well as data from prospective observational studies on extended anticoagulation for $\mathrm{CT}$, have become available. In the phase 3 CARAVAGGIO trial, ${ }^{27}$ apixaban was noninferior to the LMWH dalteparin for the prevention of VTE recurrence during the first 6 months of treatment, with no associated increased risk of major bleeding. In the phase 3 ADAM VTE study, ${ }^{26}$ apixaban was associated with a low rate of major bleeding. These results support the choice of apixaban for prospective assessment as extended treatment. In addition, data from studies of extended anticoagulant treatment beyond 6 months in patients with active cancer confirm that they remain at risk of both recurrent thrombosis and bleeding, with cumulative incidences of recurrent VTE per 7 to 12 months ranging from 1.4 to $8.0 \%$ and clinically relevant bleeding ranging from 1.4 to $4.9 \%$ (- Table 2)..$^{10,11,20,21,31,32}$ Interestingly, the results of the observational USCAT study demonstrate the influence of the cancer site on the benefitrisk ratio of anticoagulant treatment beyond 6 months. However, the absence of randomization in the observational studies and differences in patient populations might explain the variations in the findings across these studies. Data from specific prospective, randomized trials focusing on extended treatment are therefore still needed.

\section{Objectives of the API-CAT Study}

The aim of the API-CAT study is to assess whether a reduceddose regimen of apixaban ( $2.5 \mathrm{mg}$ bid) is noninferior to a fulldose regimen of apixaban ( $5 \mathrm{mg}$ bid) for the prevention of recurrent VTE in patients with active cancer who have completed at least 6 months of anticoagulant therapy for treating a documented index event of proximal DVT or PE. The key secondary objective is to assess whether a reduceddose regimen of apixaban $(2.5 \mathrm{mg}$ bid) is safer than a fulldose regimen of apixaban ( $5 \mathrm{mg}$ bid) in terms of clinically relevant bleeding.

\section{Study Design}

API-CAT (NCT03692065) is an international, prospective, randomized, parallel-group, double-blind, double-dummy, noninferiority clinical trial with blinded adjudication of outcome events. The study compares 12 months of treatment with a reduced-dose regimen of apixaban to a full-dose regimen of apixaban in patients with active cancer who have completed at least 6 months of anticoagulant treatment for the index VTE event ( $\mathbf{- F i g . 1}$ ). The study is planned to be conducted in approximately 160 centers in 11 countries (Austria, Belgium, Canada, France, Greece, Italy, Netherlands, Poland, Spain, Switzerland, and United Kingdom). 


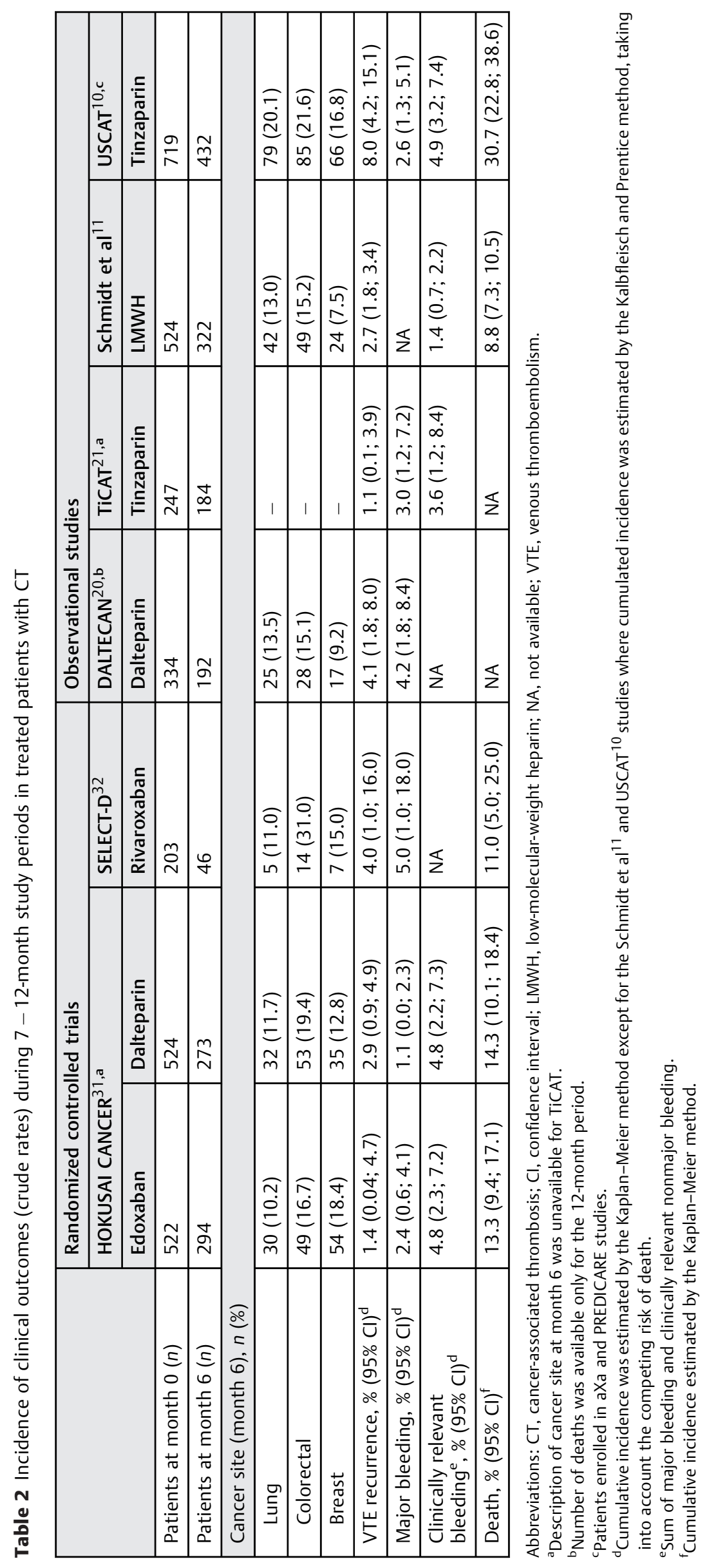




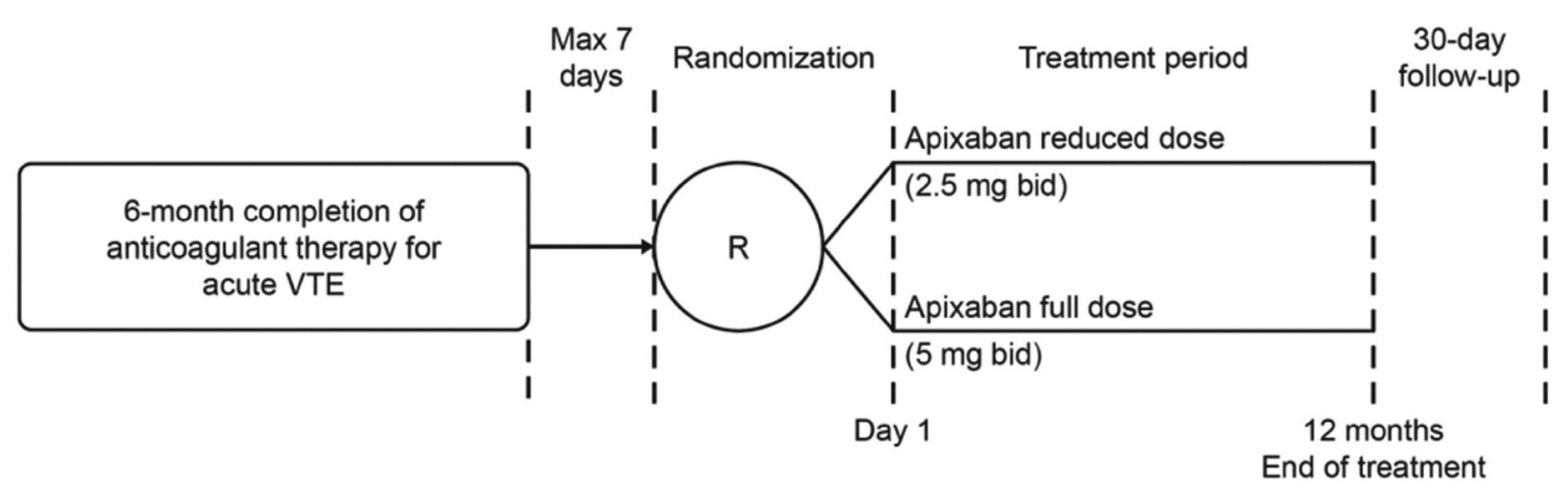

Fig. 1 Design of the randomized, double-blind, noninferiority API-CAT study. bid, twice daily; Max, maximum; mo., months; R, randomization; VTE, venous thromboembolism.

\section{Study Population and Eligibility}

Patients with active cancer who have completed at least 6 months of anticoagulant therapy for the treatment of an objectively proven symptomatic or incidental VTE, and who have not developed an objectively documented recurrence of VTE after the index event, are eligible for enrolment in the study. Documented index VTE is defined as symptomatic or incidental proximal lower-limb, iliac, and/or inferior vena cava DVT, or symptomatic or incidental PE in a segmental or larger pulmonary artery. Patients with any cancer diagnosed histologically (other than basal-cell or squamous-cell carcinoma of the skin, primary brain tumor, or intracerebral metastasis) are eligible. Active cancer is defined as the presence of measurable disease and/or ongoing (or planned) chemotherapy, radiotherapy, hormone therapy, targeted therapy, or immunotherapy at inclusion. ${ }^{33}$ Anticoagulant therapy for the index event includes any appropriate anticoagulant indicated for VTE treatment. Detailed eligibility criteria are listed in -Supplementary Table S1 (available in the online version).

\section{Rationale for the Type of Cancer Studied}

We chose to include a population with various types of cancer to ensure that the results are applicable to most patients with CT. Indeed, although some heterogeneity has been described in the respective rates of recurrence and bleeding across different cancers, most patients with CT have a reduced risk of recurrent VTE during anticoagulant treatment after 6 months whereas the risk of bleeding remains high. Furthermore, even if the prognosis of patients with CT remains poor, the substantial progress made in recent years, even in advanced disease, will make the study results applicable to these patients. Finally, even if the rates of recurrence and bleeding vary, there are no data documenting a difference in the magnitude of the treatment effect across these types of cancer.

\section{Study Treatments and Treatment Allocation}

After completing at least 6 months of anticoagulant therapy with a LMWH, DOAC, or vitamin K antagonist (VKA), and after written informed consent is obtained, patients are randomized in a double-blind fashion (1:1 ratio) to receive either a reduced-dose regimen of apixaban ( $2.5 \mathrm{mg}$ bid) or a full-dose regimen of apixaban ( $5 \mathrm{mg}$ bid) for 12 months.

Randomization of eligible patients is performed centrally using an interactive web response system. Randomization is stratified by center, type of disease treated (PE with or without DVT or isolated proximal DVT), and site of cancer (breast, prostate, colorectal, lung, other). Patients randomized to the apixaban reduced-dose group receive an apixaban $2.5 \mathrm{mg}$ tablet bid and a placebo of apixaban $5 \mathrm{mg}$ tablet bid for 12 months. Patients randomized to the apixaban fulldose group receive a placebo tablet of apixaban $2.5 \mathrm{mg}$ bid and an apixaban $5 \mathrm{mg}$ tablet bid for 12 months.

If a VKA was used at the time of inclusion in the trial, an international normalized ratio of $\leq 2$ must be documented before initiation of the study drug.

\section{Rationale for Treatment Strategies}

According to guideline recommendations, a placebo in patients completing at least 6 months of anticoagulant therapy and having active cancer was not considered to be an ethical reference treatment. ${ }^{16,17}$ Given the difficulty for patients to accept extended treatment with injections of LMWH, as observed in the ALICAT study, ${ }^{34}$ LONGHEVA study (NCT01164046), and STEPCAT study (NCT02752607), which were terminated early due to slow enrolment, we assumed that an oral treatment would have a better compliance than LMWH. The results of the AMPLIFY extension study showing promising results of the apixaban $2.5 \mathrm{mg}$ dosage, compared with the $5 \mathrm{mg}$ dosage, in terms of recurrence ( $1.7 \%$ in both apixaban groups) and major bleedings $(0.2$ and $0.1 \%$ in the $2.5 \mathrm{mg}$ and in the $5 \mathrm{mg}$ apixaban group, respectively), and reassuring data in the subgroup of patients with cancer, made us consider the opportunity to test the $2.5 \mathrm{mg}$ bid dosage against the $5 \mathrm{mg}$ bid dosage after completing the first 6 months of treatment. ${ }^{29}$ The recent evidence that apixaban $5 \mathrm{mg}$ bid is a viable therapeutic option during the first 6 months of CT treatment further supports the use of this regimen as a comparator beyond 6 months. ${ }^{27}$ 


\section{Rationale for Stratification on Type of Cancer}

The main purpose of randomization is to avoid bias by distributing patient characteristics that may influence outcome randomly between treatment groups so that any difference in outcome can be explained only by the treatment. These characteristics may be demographic, such as age, or prognostic factors. Previous studies suggested an influence of cancer site on the benefit-risk ratio of the anticoagulant treatment. ${ }^{10,22}$ The choice of a stratified randomization on the type of cancer was done to prevent imbalance between treatment groups for a known factor that may influence prognosis or treatment responsiveness in terms of efficacy and safety.

\section{Study Outcomes}

The primary efficacy outcome is a composite of adjudicated recurrent symptomatic VTE (proximal DVT and/or distal DVT and/or symptomatic PE and/or upper limb or central venous catheter thrombosis) or incidental VTE (proximal DVT or PE), or death due to PE during the 12-month treatment period (-Supplementary Table S2, available in the online version). Incidental VTE is defined as proximal DVT or PE incidentally detected by imaging when a patient undergoes imaging studies as a standard of care for the management of his or her malignancy or for any reason other than a suspicion of VTE.

The key secondary outcome is a composite of adjudicated major or clinically relevant nonmajor bleeding during the 12month treatment period (- Supplementary Table S2, available in the online version). Major bleeding is defined according to the International Society on Thrombosis and Haemostasis. ${ }^{35}$ Clinically relevant nonmajor bleeding is defined as acute clinically overt bleeding that needs specific medical attention or care but does not meet the criteria for major bleeding (-Supplementary Table S2, available in the online version).

Other outcomes are recurrent symptomatic VTE, VTE related-death, all-cause death, major bleeding, and the composite of recurrent symptomatic VTE, VTE-related death, allcause death, or major bleeding.

All suspected occurrences or recurrences of VTE, causes of deaths, and suspected episodes of bleeding during the study period are adjudicated by an independent central adjudication committee whose members are unaware of treatment allocation.

\section{Surveillance and Follow-Up}

The study requires the following scheduled visits: enrolment, 1 month, 3 months, 6 months, 9 months, 12 months, and 13 months after randomization (-Fig. $\mathbf{1}$ and - Table $\mathbf{3}$ ). Additional visits are performed if new symptoms and/or signs of VTE occur during the study period. Clinical examination and objective tests are performed if the patient develops symptoms or signs suggestive of recurrent VTE.

\section{Statistical Considerations}

\section{Sample Size Determination}

The clinical hypothesis of the API-CAT study is that a reduceddose regimen of apixaban ( $2.5 \mathrm{mg}$ bid) is noninferior to a fulldose regimen of apixaban ( $5 \mathrm{mg}$ bid) regarding the risk of recurrent VTE, assuming that the efficacies of the reduced dose and of the full dose of apixaban are superimposed (relative risk =1). Assuming a $4 \%$ annual incidence rate of recurrent VTE based on previous studies, ${ }^{20,22,29}$ a possible dropout rate of $10 \%$, and that apixaban $2.5 \mathrm{mg}$ bid will be considered as noninferior to apixaban $5 \mathrm{mg}$ bid if the upper limit of the two-sided $95 \% \mathrm{CI}$ of the relative hazard ratio (HR) is less than 2.0, 1,722 patients (65 events, 861 patients per group) will be required to have $80 \%$ power to show noninferiority of apixaban $2.5 \mathrm{mg}$ bid with a one-sided type I error of $2.5 \%$.

The sample size will also provide sufficient power to demonstrate a clinically significant reduction in the key secondary endpoint (major or clinically relevant nonmajor bleeding). Based on previous studies, ${ }^{20,22,29}$ we assumed a $6 \%$ frequency of major or clinically relevant nonmajor bleeding with apixaban $5 \mathrm{mg}$ bid in this population at high risk of bleeding. With an overall sample size of 1,640 patients (65 events, 820 patients per group), the study would have $80 \%$ power to show a $50 \%$ risk reduction using apixaban $2.5 \mathrm{mg}$ bid, with a two-sided type I error of $5 \%$ (and a possible dropout rate of $10 \%)$. All calculations for sample size estimation were performed with NQUERY 7 software (Statistical Solutions, Boston, United States).

As the sample size determination did not take into account a possible competing risk for death, a review of the blinded aggregate rate for the primary efficacy endpoint as well as the incidence of patients with missing data (patients who did not have a primary efficacy endpoint for competing risk) will be provided to the steering committee after $80 \%$ of subjects have been randomized (i.e., 1,400 patients). The sample size may be adjusted to provide sufficient power for the noninferiority test on the primary efficacy endpoint. This blinded review will be performed by an independent statistician.

\section{Statistical Analysis}

To determine the noninferiority of apixaban $2.5 \mathrm{mg}$ bid versus apixaban $5 \mathrm{mg}$ bid, the primary analysis will be performed in the intent-to-treat (ITT) population. The ITT population will include all randomized patients, each patient remaining in the group to which they were assigned, regardless of the treatment received. As the main comparison is a noninferiority test, the primary analysis will also be performed in the per-protocol population, including all randomized patients who received at least one dose of the study medication and without any major protocol violations or deviations (i.e., not meeting the inclusion criteria, and study treatment interrupted for $>30$ consecutive days), as recommended by the European Medicines Agency guidelines. ${ }^{36}$ The per-protocol population will be defined in the statistical analysis plan before unblinding of the data. For unresolved 
Table 3 Flowchart/patient follow-up summary and distinction between procedures associated with usual care and procedures performed because of the API-CAT study protocol

\begin{tabular}{|c|c|c|c|c|c|c|c|}
\hline & $\begin{array}{l}\text { Baseline } \\
\text { visit }^{\text {a }}\end{array}$ & $\begin{array}{l}\text { Week } \\
4^{b} \pm 15 \\
\text { days }\end{array}$ & $\begin{array}{l}\text { Month } \\
3 \pm 15 \\
\text { days }\end{array}$ & $\begin{array}{l}\text { Month } \\
6 \pm 15 \\
\text { days }\end{array}$ & $\begin{array}{l}\text { Month } \\
9 \pm 15 \\
\text { days }\end{array}$ & $\begin{array}{l}\text { Month } 12 \\
\text { (end of } \\
\text { treatment } \\
\text { visit } \pm 15 \text { days }\end{array}$ & $\begin{array}{l}\text { Month } 13 \\
\text { (30-day } \\
\text { posttreatment } \\
\text { visit } \pm 15 \text { days }\end{array}$ \\
\hline Informed consent $^{\mathrm{C}}$ & $x$ & & & & & & \\
\hline Inclusion/exclusion criteriac ${ }^{c}$ & $x$ & & & & & & \\
\hline Randomization $^{c}$ & $x$ & & & & & & \\
\hline Medical history ${ }^{c}$ & $\mathrm{X}$ & & & & & & \\
\hline Physical examination & $x$ & $x$ & $x$ & $x$ & $x$ & $x$ & \\
\hline Height, weight & $x$ & & & & & & \\
\hline Vital signs & $x$ & $x$ & $x$ & $x$ & $x$ & $x$ & \\
\hline Documentation of index event & $x$ & & & & & & \\
\hline Adverse event assessment ${ }^{\mathrm{c}}$ & & $x$ & $x$ & $x$ & $x$ & $x$ & $x$ \\
\hline Outcome assessment $^{c}$ & & $x$ & $x$ & $\bar{x}$ & $x$ & $x$ & $\mathrm{x}$ \\
\hline Clinical laboratory tests $^{\mathrm{d}}$ & $x$ & $x$ & $x$ & $x$ & $x$ & $x$ & \\
\hline Urinary pregnancy test $^{\mathrm{C}}$ & $x$ & $x$ & $x$ & $x$ & $x$ & $x$ & \\
\hline Assess medication use ${ }^{c}$ & & $x$ & $x$ & $x$ & $x$ & $\mathrm{x}$ & \\
\hline $\begin{array}{l}\text { Assess concomitant } \\
\text { medication use }\end{array}$ & $x$ & $x$ & $x$ & $x$ & $x$ & $x$ & $x$ \\
\hline $\begin{array}{l}\text { Investigational } \\
\text { product dispensed }\end{array}$ & $x$ & & $x$ & $x$ & $x$ & & \\
\hline
\end{tabular}

${ }^{a}$ Clinical laboratory test results performed within 2 weeks of the date of this visit can be used.

${ }^{\mathrm{b}}$ For centers at which standard care does not require the patient to attend a hospital appointment 4 weeks after randomization, the investigator should prescribe the 4-week laboratory tests (the results will be sent to the investigator as usual), and give women of child-bearing potential a urinary pregnancy test (when required) at the baseline visit. The patient (or their relative) will be contacted by telephone for the 4-week visit to ask about any suspicion of VTE recurrence, bleeding, death or adverse event since randomization, and assess study medication use and concomitant medication use.

"Visits or procedures added because of "research."

${ }^{d}$ Clinical laboratory tests done at baseline visit: hematocrit, hemoglobin, blood cell count, platelet count, international normalized ratio, and activated partial prothrombin time in case of previous treatment with vitamin $\mathrm{K}$ antagonist, albumin, blood urea nitrogen (urea), creatinine, creatinine clearance, potassium, sodium, alkaline phosphatase, alanine transaminase, aspartate aminotransferase, direct bilirubin, total bilirubin, and gamma-glutamyl transferase. Clinical laboratory tests at other visits: hemoglobin, platelet count, creatinine, creatinine clearance, alanine transaminase, aspartate aminotransferase, and direct bilirubin.

cases, inclusion or exclusion in the per-protocol population will be decided by the blinded review committee.

A time-to-event analysis will be performed to estimate the risk of recurrent VTE in the two groups. As all patients have active cancer and thereby are at high risk of death due to cancer progression, a time-to-event analysis will be performed using the Fine and Gray regression model with stratification variables as covariates to take into account the competing risk of death. ${ }^{37,38}$ Treatment effect will be estimated by the HR and its 95\% two-sided CI and overall significance will be set at the two-sided $\alpha=0.05$ level.

The analysis of the primary endpoint will be done by considering the time from randomization to the first recurrent VTE (outcome) or to death for a cause other than VTErelated (competing event) or to the last known follow-up date if neither a recurrent VTE nor a competing event occurs within the 12-month follow-up period (censored time). The statistical analysis for the primary efficacy outcome will be conducted on the ITT and per-protocol populations, according to European Medicines Agency recommendations. ${ }^{39}$
Superiority of the reduced dose of apixaban ( $2.5 \mathrm{mg}$ bid) in comparison with the full dose of apixaban ( $5 \mathrm{mg}$ bid) will also be tested if noninferiority is demonstrated.

Superiority of the key secondary endpoint (major or clinically relevant nonmajor bleeding) will be tested in a prespecified hierarchical strategy (i.e., only if noninferiority is demonstrated for the primary endpoint). For the key secondary endpoint and other safety endpoints, the statistical analysis will be conducted on the ITT population.

Detailed methodology for statistical analyses of the data collected in this trial will be documented in a statistical analysis plan, which will be finalized, dated, and maintained by the sponsor before unblinding the data. No interim analysis of efficacy is planned.

\section{Rationale for Using a Competing Risk Analysis}

In clinical studies, time-to-event statistical approaches such as the Kaplan-Meier method are typically used to account for 
unequal follow-up times (i.e., patients who die or drop out before study completion). These survival analysis methods were originally developed to describe all-cause death in the presence of a loss to follow-up that is independent of the study outcome, and are now widely used to describe outcomes other than all-cause death, especially in the field of oncology. However, in the presence of a significant related competing risk such as death, the standard method of Kaplan-Meier may lead to biased results. In patients with CT, with a mortality rate exceeding $30 \%$ at 6 months, ${ }^{40,41}$ increasing to approximately $45 \%$ at 12 months, ${ }^{24}$ mostly due to the cancer progression, the standard statistical method of Kaplan-Meier to evaluate time to recurrence is not suitable and overestimates the cumulative incidence of VTE by failing to account for the competing risk of death. In the competitive-risk analysis, competing events are handled as actual events rather than censoring observations and take into account that a patient's death prevents the occurrence of VTE. Thereby, the probability of experiencing a recurrence of VTE is adjusted for the competing risk of death that occurred before the recurrence of VTE.

\section{Rationale for Using a Hierarchical Sequential Testing Strategy}

The objectives of the API-CAT study are (1) to demonstrate that a reduced-dose regimen of apixaban is noninferior to a full-dose regimen of apixaban, and (2) to assess whether a reduced-dose regimen of apixaban is safer than a full-dose regimen of apixaban. In this context of two coprimary objectives, the problem arises of the multiplicity of tests and the inflation of type I error. In addition, the second objective makes sense only if the first is reached. A hierarchical testing strategy is one of the solutions to control type I error at a rate of $5 \%$. Indeed, at the time of the analysis, the criteria are considered one after the other, sequentially, following the pre-established hierarchy. It will be possible to conclude that the effect has been demonstrated for all of the first criteria that are statistically significant at the usual level of $5 \%$ up to the first nonsignificant test. The advantage of this approach is that it may be possible (depending on the results obtained) to conclude that the effect has been demonstrated simultaneously on several criteria without inflation of the $\alpha$ type I error.

\section{Study Organization}

\section{Ethical and Regulatory Considerations}

The study is being conducted according to globally accepted standards of the International Conference on Harmonization Good Clinical Practice, and in agreement with the latest revision of the ethical principles laid down in the Declaration of Helsinki and in keeping with applicable local regulations (including European Directive 2001/20/EC). Protocol, amendments, and the proposed informed consent are reviewed and approved by the institutional review board or independent ethics committee or research ethics board at each site or country according to the national legislation.
The study is sponsored by the Assistance Publique des Hôpitaux de Paris (AP-HP, France). The study is coordinated by the Clinical Research and Innovation Unit of the AP-HP and the steering committee of the study. Data are collected and maintained using the CleanWeb electronic data capture system and will be analyzed by the Clinical Research Unit of the University Hospital of Saint-Etienne (France) under the supervision of the steering committee members.

\section{Study Monitoring and Adverse Event Reporting}

The study is conducted using the CleanWeb electronic data capture system and validated according to the most recent Good Clinical Practice guidelines. Data collected during baseline and follow-up visits and occurrence of any adverse events, including serious adverse events, are recorded in the electronic case report form, which enables management of all the data in a single system, improving accuracy, visibility, and data integrity. Any suspected unexpected serious adverse reaction must also be declared electronically using the EudraVigilance European adverse drug reactions database managed by the European Medicines Agency. All the adverse events are coded using the Medical Dictionary for Regulator Activities. The data safety monitoring board (DSMB) oversees the safety of the study subjects by periodically assessing the safety and efficacy of the trial therapy, and monitors the integrity and validity of the data collected and the conduct of the trial.

\section{Study Committees}

The steering committee comprises the principal investigator, scientific director, methodologist, a group of clinical experts in thrombosis or oncology representing all participating countries, and the study statistician. This committee has the final responsibility for the study design and oversight as well as the verification and analyses of all the study data. All members of the steering committee will have access to the study data, contribute to the interpretation of the results, approve the final version of the manuscript, vouch for the accuracy and completeness of the data reported and the fidelity of the article to the study protocol, and make the decision to submit the manuscript for publication.

An independent central adjudication committee, whose members are unaware of treatment allocation, reviews and adjudicates all suspected recurrences of VTE, cause of deaths, and suspected episodes of bleeding occurring during the study period. The committee comprises physicians with experience in vascular medicine, oncology, and radiology. An independent central adjudication committee charter, consistent with the study protocol, details the diagnostic criteria for all adjudicated clinical events.

An independent DSMB, whose members are not involved in the conduct of the study, periodically assesses the safety and efficacy of the study therapy and monitors the integrity and validity of the data collected and the conduct of the study. A DSMB charter was provided to the board members, which was finalized before the first patient was enrolled. The DSMB comprises five expert clinicians with experience in the conduct and monitoring of clinical trials. 


\section{Anticipated Results and Implications for Clinical Practice}

The ongoing prospective randomized EVE study ${ }^{42}$ is focusing on the safety (major bleeding endpoint including fatal bleeding or clinically relevant nonmajor bleedings) of apixaban $2.5 \mathrm{mg}$ versus $5 \mathrm{mg}$ bid for extended treatment in patients with CT. Unlike the API-CAT study, the inclusion criteria comprise upper extremity DVT and splanchnic or cerebral vein thrombosis.

The API-CAT trial has the potential to propose an effective and safe regimen for secondary prevention of VTE beyond 6 months in patients with CT.

\section{What is known about this topic?}

- Cancer patients with venous thromboembolism (VTE) remain at increased risk of recurrence and bleeding after the initial 6-month anticoagulant treatment period.

- For the treatment of VTE in patients with cancer, a fulldose regimen of a direct oral anticoagulant (DOAC) appears as effective and as safe as low-molecularweight heparin, but no data are currently available on the reduced-dose regimen of DOAC after the initial 6-month treatment period.

\section{What does this paper add?}

- This paper details the design of the ongoing API-CAT study, a prospective, randomized, controlled, doubleblind, noninferiority trial that compares a reduced dose versus a full dose of apixaban for 12 months in patients with active cancer who have already completed 6 months of anticoagulant treatment for VTE.

- This trial has the potential to demonstrate that a reduced dose of apixaban does not increase the risk of VTE recurrence while decreasing the risk of bleeding.

\section{Funding}

Funding for the study is provided by the BMS-Pfizer Alliance. Tablets of apixaban are provided by BristolMyers Squibb and are packaged under AP-HP's responsibility. Bristol-Myers Squibb and Pfizer did not have any role in the study design, study conduction, data collection, or analysis.

\section{Conflict of Interest}

I.M. reports an independent research grant to the sponsor (AP-HP) from BMS/Pfizer. I.M. has received a research grant from Leo Pharma, has been a speaker for BMS and Leo Pharma, and has participated in advisory boards for BMS and Bayer. G.A.: no disclosure was declared by the author. C.A. has received honoraria for lectures from
Bayer, BKS, Pfizer, Sanofi, and Daiichi Sankyo, and has participated in advisory boards for Bayer, BMS, Pfizer, and Sanofi. C.B. has received consulting fees/honoraria from Bayer Healthcare, Daiichi Sankyo, and BMS. A.B. has nothing to disclose. M.C. reports research funding from BMS, Pfizer, and Leo Pharma, and honoraria from BMS, Pfizer, Sanofi, Bayer, Leo Pharma, Servier, and Valeo; all payments were made to the institution. C.C. has received consulting fees from Leo Pharma. A.T.C.: no disclosure was declared by the author. P.G. has received honoraria and support for attending meetings and/or travel from Leo Pharma and Bayer. M.V.H. reports grants from ZonMW Dutch Healthcare Fund, and grants and personal fees to the hospital from Boehringer Ingelheim, BMS/Pfizer, Bayer Healthcare, Aspen, and Daiichi Sankyo, all outside the submitted work. F.A.K. reports research grants from Bayer, BMS, Boehringer Ingelheim, Daiichi Sankyo, MSD, The Netherlands Organization for Health Research and Development, Actelion, the Dutch Heart Foundation, and the Dutch Thrombosis Association, all outside the submitted work. J.J.L.-N. has received honoraria for: lectures from Bayer, Pfizer and Rovi; educational events from Leo Pharma; lectures and support for attending meetings and travel from Bayer, Boehringer Ingelheim, BMS, Pfizer, Leo Pharma, Rovi, and Sanofi; and advisory board participation from Pfizer and Techdow. A.M. has received honoraria from Bayer, BMS, Leo Pharma, Rovi, and Pfizer, and support for attending meetings and/or travel from Bayer and BMS, and has participated in a data safety monitoring board or an advisory board for Bayer, BMS, and Leo Pharma. D.M. has received consulting fees from Leo Pharma, Pfizer, and BMS, and honoraria and support for attending meetings and/or travel from Leo Pharma and Pfizer. O.M. has received consulting fees from AstraZeneca, Blueprint Medicines, Boehringer-Ingelheim, BristolMyers Squibb, Eli Lilly, Ipsen, Merck Sharpe \& Dohme, Pfizer, Roche, Servier, and Vifor Pharma, payment or honoraria for lectures, presentations, speakers bureaus, manuscript writing or educational events from Blueprint Medicines, Eli-Lilly, Pfizer, Roche, and support for attending meetings and/or travel from Amgen and Roche, and has participated on a data safety monitoring board or advisory board for Blueprint Medicines, Boehringer-Ingelheim; and holds stock or stock options in Amplitude Surgical and Ipsen Transgene. M.M. has received an unrestricted grant for research from Sanofi, Leo Pharma, and Rovi, consulting fees from Sanofi and Bristol, and honoraria for lectures from Sanofi and Pfizer. M.R. has received honoraria for lectures from Bayer, Pfizer, and Biomérieux. C.M.S. has participated in a data safety monitoring board for the API-CAT study. K.S. has received personal fees from Roche, BMS, and MSD, and institutional fees from Amgen. S.S. has received honoraria for lectures from Bayer, BMS, Pfizer, Angelini, Janssen-Cilag, and Roche, and support for attending meetings and/or travel from BMS. A.T. has received consulting fees, payment or honoraria for lectures, presentations, speakers bureaus, manuscript writing or educational events, and participated on an advisory 
board for Bayer and Pfizer. P.V. is co-holder of the Bayer Chair in Cardiovascular Medicine at the University of Leuven. He has received consulting fees from Bayer, Daiichi Sankyo, Portola, and Anthos Pharmaceuticals, and honoraria from Bayer, Daiichi Sankyo, BMS, Pfizer, Boehringer, and Leo Pharma. E.V. has contracts for consulting in statistics with Pfizer, Novartis, Boehringer, Hexacath, Amgen, and Abbott. He has received fees for consulting in statistics from Pfizer, Novartis, Boehringer, Hexacath, Amgen, and Abbott, and honoraria for lectures on statistics from Amgen. T.-F.W. has received a research grant from Leo Pharma, and has participated in advisory boards for Pfizer and Servier. G.M. is deceased. S.L. has received grants from Leo Pharma, grants and consulting fees from Bayer Healthcare, and speaker fees from BMS, Pfizer, Merck Serono. The other authors report no conflicts of interest.

\section{Acknowledgment}

Dr. Sophie Rushton-Smith (MedLink Healthcare Communications, London) provided editorial assistance in the preparation of the manuscript (limited to editing for style, referencing, and figure and table editing) and was funded by the authors.

\section{References}

1 Timp JF, Braekkan SK, Versteeg HH, Cannegieter SC. Epidemiology of cancer-associated venous thrombosis. Blood 2013;122(10): $1712-1723$

2 Mulder FI, Horváth-Puhó E, van Es N, et al. Venous thromboembolism in cancer patients: a population-based cohort study. Blood 2021;137(14):1959-1969

3 Bray F, Ferlay J, Soerjomataram I, Siegel RL, Torre LA, Jemal A. Global cancer statistics 2018: GLOBOCAN estimates of incidence and mortality worldwide for 36 cancers in 185 countries. CA Cancer J Clin 2018;68(06):394-424

4 Siegel RL, Miller KD, Jemal A. Cancer statistics, 2019. CA Cancer J Clin 2019;69(01):7-34

5 Quaresma M, Coleman MP, Rachet B. 40-year trends in an index of survival for all cancers combined and survival adjusted for age and sex for each cancer in England and Wales, 1971-2011: a population-based study. Lancet 2015;385(9974):1206-1218

6 Rosenberg JE, Hoffman-Censits J, Powles T, et al. Atezolizumab in patients with locally advanced and metastatic urothelial carcinoma who have progressed following treatment with platinumbased chemotherapy: a single-arm, multicentre, phase 2 trial. Lancet 2016;387(10031):1909-1920

7 Schachter J, Ribas A, Long GV, et al. Pembrolizumab versus ipilimumab for advanced melanoma: final overall survival results of a multicentre, randomised, open-label phase 3 study (KEYNOTE-006). Lancet 2017;390(10105):1853-1862

8 Balar AV, Galsky MD, Rosenberg JE, et al; IMvigor210 Study Group. Atezolizumab as first-line treatment in cisplatin-ineligible patients with locally advanced and metastatic urothelial carcinoma: a single-arm, multicentre, phase 2 trial. Lancet 2017;389 (10064):67-76

9 Motzer RJ, Tannir NM, McDermott DF, et al; CheckMate 214 Investigators. Nivolumab plus ipilimumab versus sunitinib in advanced renal-cell carcinoma. N Engl J Med 2018;378(14): 1277-1290

10 Mahé I, Plaisance L, Chapelle C, et al. Long-term treatment of cancer-associated thrombosis (CAT) beyond 6 months in the medical practice: USCAT, a 432-patient retrospective non-interventional study. Cancers (Basel) 2020;12(08):2256

11 Schmidt RA, Al Zaki A, Desilet N, et al. Patient characteristics and long-term outcomes beyond the first 6 months after a diagnosis of cancer-associated venous thromboembolism. Thromb Res 2020; 188:106-114

12 Mahé I, Mayeur D, Krakowski I. Management of venous thromboembolism in cancer patients: the economic burden of hospitalizations. Support Care Cancer 2016;24(10):4105-4112

13 Cohoon KP, Ransom JE, Leibson CL, et al. Direct medical costs attributable to cancer-associated venous thromboembolism: a population-based longitudinal study. Am J Med 2016;129:1000. e15-1000.e25

14 Cohen AT, Katholing A, Rietbrock S, Bamber L, Martinez C. Epidemiology of first and recurrent venous thromboembolism in patients with active cancer. A population-based cohort study. Thromb Haemost 2017;117(01):57-65

15 van der Hulle T, den Exter PL, van den Hoven P, et al. Cohort study on the management of cancer-associated venous thromboembolism aimed at the safety of stopping anticoagulant therapy in patients cured of cancer. Chest 2016;149(05):1245-1251

16 Kearon C, Akl EA, Ornelas J, et al. Antithrombotic therapy for VTE disease: CHEST guideline and expert panel report. Chest 2016; 149(02):315-352

17 Farge D, Frere C, Connors JM, et al; International Initiative on Thrombosis and Cancer (ITAC) advisory panel. 2019 International clinical practice guidelines for the treatment and prophylaxis of venous thromboembolism in patients with cancer. Lancet Oncol 2019;20(10):e566-e581

18 Key NS, Khorana AA, Kuderer NM, et al. Venous thromboembolism prophylaxis and treatment in patients with cancer: ASCO clinical practice guideline update. J Clin Oncol 2020;38(05): 496-520

19 Lyman GH, Carrier M, Ay C, et al. American Society of Hematology 2021 guidelines for management of venous thromboembolism: prevention and treatment in patients with cancer. Blood Adv 2021;5(04):927-974

20 Francis CW, Kessler CM, Goldhaber SZ, et al. Treatment of venous thromboembolism in cancer patients with dalteparin for up to 12 months: the DALTECAN Study. J Thromb Haemost 2015;13(06): 1028-1035

21 Jara-Palomares L, Solier-Lopez A, Elias-Hernandez T, et al. Tinzaparin in cancer associated thrombosis beyond 6months: TiCAT study. Thromb Res 2017;157:90-96

22 Mahé I, Chidiac J, Bertoletti L, et al; RIETE investigators. The clinical course of venous thromboembolism may differ according to cancer site. Am J Med 2017;130(03):337-347

23 Schaefer JK, Li M, Wu Z, et al. Anticoagulant medication adherence for cancer-associated thrombosis: a comparison of LMWH to DOACs. J Thromb Haemost 2021;19(01):212-220

24 Raskob GE, van Es N, Verhamme P, et al; Hokusai VTE Cancer Investigators. Edoxaban for the treatment of cancer-associated venous thromboembolism. N Engl J Med 2018;378(07):615-624

25 Young AM, Marshall A, Thirlwall J, et al. Comparison of an oral factor Xa inhibitor with low molecular weight heparin in patients with cancer with venous thromboembolism: results of a randomized trial (SELECT-D). J Clin Oncol 2018;36(20):2017-2023

26 McBane RD II, Wysokinski WE, Le-Rademacher JG, et al. Apixaban and dalteparin in active malignancy-associated venous thromboembolism: the ADAM VTE trial. J Thromb Haemost 2020;18(02): 411-421

27 Agnelli G, Becattini C, Meyer G, et al; Caravaggio Investigators. Apixaban for the treatment of venous thromboembolism associated with cancer. N Engl J Med 2020;382(17):1599-1607

28 Giustozzi M, Agnelli G, Del Toro-Cervera J, et al. Direct oral anticoagulants for the treatment of acute venous thromboembolism associated with cancer: a systematic review and metaanalysis. Thromb Haemost 2020;120(07):1128-1136 
29 Agnelli G, Buller HR, Cohen A, et al; AMPLIFY-EXT Investigators. Apixaban for extended treatment of venous thromboembolism. N Engl J Med 2013;368(08):699-708

30 Agnelli G, Buller HR, Cohen A, et al; AMPLIFY Investigators. Oral apixaban for the treatment of acute venous thromboembolism. N Engl J Med 2013;369(09):799-808

31 Di Nisio M, van Es N, Carrier M, et al. Extended treatment with edoxaban in cancer patients with venous thromboembolism: a post-hoc analysis of the Hokusai-VTE Cancer study. J Thromb Haemost 2019;17(11):1866-1874

32 Marshall A, Levine M, Hill C, et al. Treatment of cancer-associated venous thromboembolism: 12-month outcomes of the placebo versus rivaroxaban randomization of the SELECT-D Trial (SELECTD: 12m). J Thromb Haemost 2020;18(04):905-915

33 Kearon C, Ageno W, Cannegieter SC, Cosmi B, Geersing GJ, Kyrle PASubcommittees on Control of Anticoagulation, and Predictive and Diagnostic Variables in Thrombotic Disease. Categorization of patients as having provoked or unprovoked venous thromboembolism: guidance from the SSC of ISTH. J Thromb Haemost 2016; 14(07):1480-1483

34 Noble SI, Nelson A, Fitzmaurice D, et al. A feasibility study to inform the design of a randomised controlled trial to identify the most clinically effective and cost-effective length of Anticoagulation with Low-molecular-weight heparin In the treatment of Cancer-Associated Thrombosis (ALICAT). Health Technol Assess 2015;19(83):vii-xxiii, 1-93

35 Schulman S, Kearon CSubcommittee on Control of Anticoagulation of the Scientific and Standardization Committee of the International Society on Thrombosis and Haemostasis. Definition of major bleeding in clinical investigations of antihemostatic medicinal products in non-surgical patients. J Thromb Haemost 2005;3(04):692-694

36 The European Agency for the Evaluation of Medicinal Products. Committee for Proprietary Medicinal Products (CPMP). Points to consider on switching between superiority and non-inferiority. Accessed July 1, 2021 at: https://www.ema.europa.eu/en/documents/scientific-guideline/points-consider-switching-betweensuperiority-non-inferiority_en.pdf

37 Campigotto F, Neuberg D, Zwicker JI. Biased estimation of thrombosis rates in cancer studies using the method of Kaplan and Meier. J Thromb Haemost 2012;10(07):1449-1451

38 Fine JP, Gray RJ. A proportional hazards model for the subdistribution of a competing risk. J Am Stat Assoc 1999;94:496-509

39 Committee for Proprietary Medicinal Products. Points to consider on switching between superiority and non-inferiority. Br J Clin Pharmacol 2001;52(03):223-228

40 Lee AY, Levine MN, Baker RI, et al; Randomized Comparison of Low-Molecular-Weight Heparin versus Oral Anticoagulant Therapy for the Prevention of Recurrent Venous Thromboembolism in Patients with Cancer (CLOT) Investigators. Low-molecular-weight heparin versus a coumarin for the prevention of recurrent venous thromboembolism in patients with cancer. N Engl J Med 2003;349 (02):146-153

41 Lee AYY, Kamphuisen PW, Meyer G, et al; CATCH Investigators. Tinzaparin vs warfarin for treatment of acute venous thromboembolism in patients with active cancer: a randomized clinical trial. JAMA 2015;314(07):677-686

42 McBane RD II, Loprinzi CL, Ashrani A, et al. Extending venous thromboembolism secondary prevention with apixaban in cancer patients: The EVE trial. Eur J Haematol 2020;104(02):88-96 